\title{
A Scintillating Fiber Beam Halo Detector for Heavy Ion Beam Diagnostics
}

M.A. McMahan, A. Assang, S. Herr, F. McCormack, G. Krebs, and B. Feinberg

Accelerator and Fusion Research Division, Lawrence Berkeley Laboratory

1 Cyclotron Road, Berkeley, CA 94720

\section{Abstract}

A prototype beam halo detector of scintillating fibers has been designed in order to detect beam halo problems at an early stage in the tuning process. The results of initial characterization of the fibers are presented and issues of electronics and readout discussed.

\section{INTRODUCTION}

The Bevalac at Lawrence Berkeley Laboratory (LBL), before its closure in February of 1993, was a weak-focussing synchrotron injected by the SuperHILAC linac. The Bevalac had the capability of running protons (up to $5 \mathrm{GeV}$ ) through uranium (up to $960 \mathrm{MeV} /$ nucleon). This wide range of beams and energies caused unique problems in beam diagnostics, which were exacerbated by the large size of the beam in the accelerator and the method of extraction.

A particularly nasty diagnostic problem was the measurement of beam halo, whether caused by the extraction process, scraping on apertures, or charge-changing in the poor vacuum. The halo could be several orders of magnitude less than the peak beam intensity and thus undetectable during the tuning process using the standard Bevalac wire chambers. Yet this halo could wreak havoc with experimenters' detectors, sometimes causing insurmountable background problems. In one celebrated case, a low energy heavy ion run (the worst conditions for extraction problems as well as vacuum problems), a satellite beam $14^{\circ}$ removed from the main beam made it through a wholc beam line and into delicate detectors, bypassing both collimator systems and the target assembly along the way.

Because of experiences like these, a project was embarked upon to first, characterize the beam halo and try to understand what was the predominant cause, and then, to set up a diagnostic system for beam halo which the operators could use as part of the tuning process. The idea was eventually to have halo detectors in every beam line. Initial tests were done and a prototype detector built before the closure of the Bevalac. This detector will be described in the remainder of this paper.

\section{THE HALO DETECTOR}

\section{Requirements}

The main requirement of the beam halo detector was the ability to detect a large range of beam intensities, particles, and energies. This requirement made the wire chamber system useless for beam halo. For example, Figure 1 gives the calculated energy loss as a function of energy for ions ranging from protons to gold $(\mathrm{Z}=79)$. The energy loss of an ion in a 1 $\mathrm{mm}$. scintillator ranges from $10000 \mathrm{McV}$ for gold at low energies down to near $0.1 \mathrm{MeV}$ for protons at high energies. Coupling this with a perhaps factor of 1000 in beam intensity between the main beam and the halo, one can imagine the magnitude of the problem.

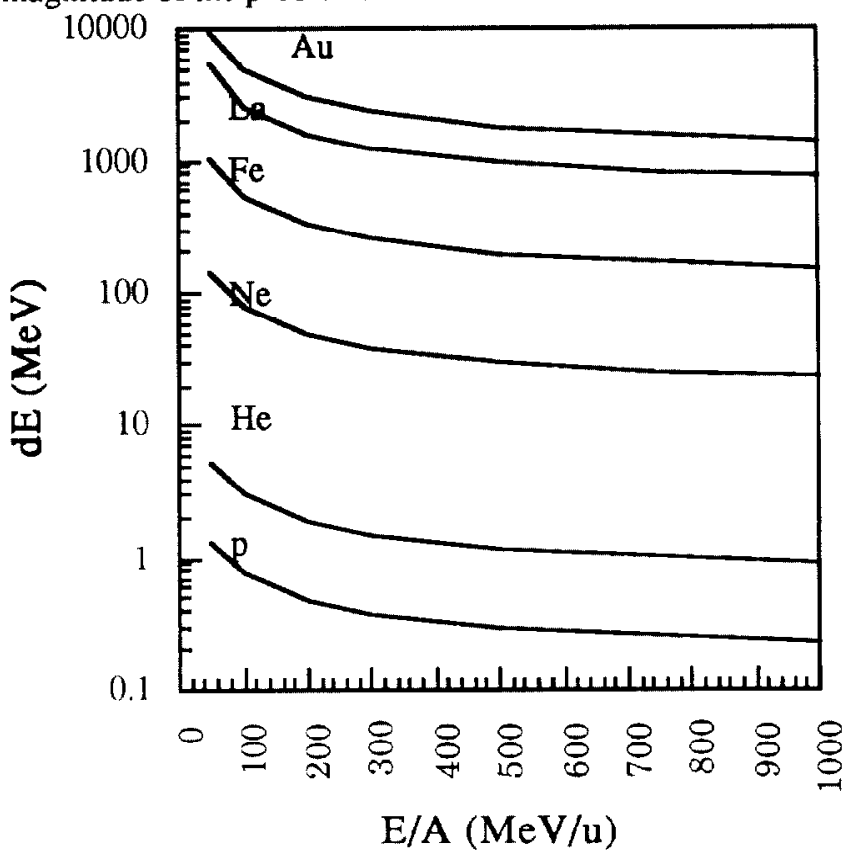

Figure 1. Calculated Energy Loss in $1 \mathrm{~mm}$. scintillating fiber

Other considerations were that the chosen detector should be a high-count rate, radiation resistant, device which would integrate well into the wire chamber readouts for tuning purposes. Plastic scintillator was settled on early as the detector material of choice because it will take relatively high rates and is less subject to radiation damage than other material, as well as being inexpensive and versatile. Recent technological advances have made a variety of plastic scintillating fiber readily available. These fibers are bcginning to be widely used in high energy physics as well as space applications. [1] Using fibers, a detcctor could be made that looked like a Bevalac wire chamber, mounting on the same hardware, but covering the larger dynamic range necessary to tune on beam halo.

The Detector

The design of the prototype detector is shown in Figure 2. Because most of the wire chambers in the beam lines have wire spacings of $6 \mathrm{~mm}$. in an 8 " beam pipe, a similar geometry was chosen for the scintillating fiber detector. Twelve fibers were used in each of two planes (horizontal and vertical) separated by approximately $1 \mathrm{~cm}$. The fibers were spaced $6 \mathrm{~mm}$. apart in the central beam region, with $12 \mathrm{~mm}$. spacing in the outer halo regions, giving a total coverage of $10 \mathrm{~cm}$. Each scintillating fiber was coupled on the edge of the mount to an optical fiber of the same composition and diameter. The coupling was done with a layer of optical 
grease. The optical fibers were brought out of the vacuum through an epoxy-filled feedthrough. For the initial tests, each fiber was coupled directly to a photomultiplier tube (PMT).

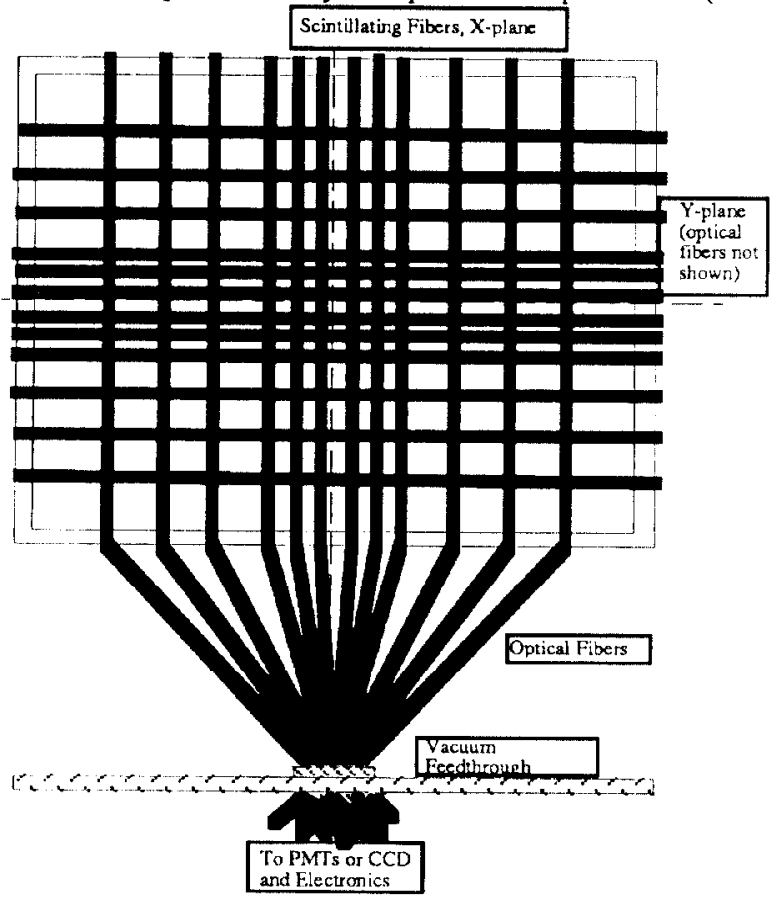

Figure 2. Prototype Detector

\section{The Fibers}

Initial tests were done with several scintillating fibers of varying diameter; some were square in cross section and some round. We settled on using $1 \mathrm{~mm}$. diameter round BCF. 10 fiber, a blue-emitting fiber with a polystyrene core and PMMA cladding made be Bicron Corporation. [2] The $1 \mathrm{~mm}$. dlameter was thick enough so that the individual fibers could be mounted in a rigid fashion. The fibers were tested with a laser beam, an electron source, and in several test runs with different beams.

Electronics and Data Acquisition

A computer acquisition system was developed for these tests based on a Macintosh FX [3] running National Instrument's Labview acquisition software [4] to read signals from standard CAMAC modules over a GPIB interface. This acquisition proved to be slow but adequate for these tests. The output from the PMTs were taken into an Charge-to-Digital Converter (QDC) and converted to a pulse height. A sample spectra is shown in Figure 3 for a $300 \mathrm{MeV} /$ nucleon Au beam passing through the fiber, with a calculated energy loss of approximately $2300 \mathrm{MeV}$. The background was negligible in these runs, consisting only of low pulse height counts in the PMTs, and easily discriminated out.

The use of individual PMTs with 24 channels of electronics added a layer of complexity to the detector that was counter to the goal of a user-friendly device for operator tuning. For the next step, it was planned to read all the fibers out into a charge-coupled device (CCD). One could then either use a frame-grabber board in the Macintosh to digitize the CCD output and convert it into a beam profile, or the CCD output could be displayed directly on a video monitor in the Control Room. Similar systems have been used in biological and space applications. [1,5] For this application a low resolution but large dynamic range $\mathrm{CCD}$ chip would be necessary. It was not clear whether an image intensifier would be needed in order to meet the dynamic range requirements. If it were, it would add significantly to the cost. In order to try to answer this question, many of the initial tests on the prototype delector were designed to get an estimate of the light output of the fibers and the transmission through the detector to the PMT. These tests and calculations are described in the next section.

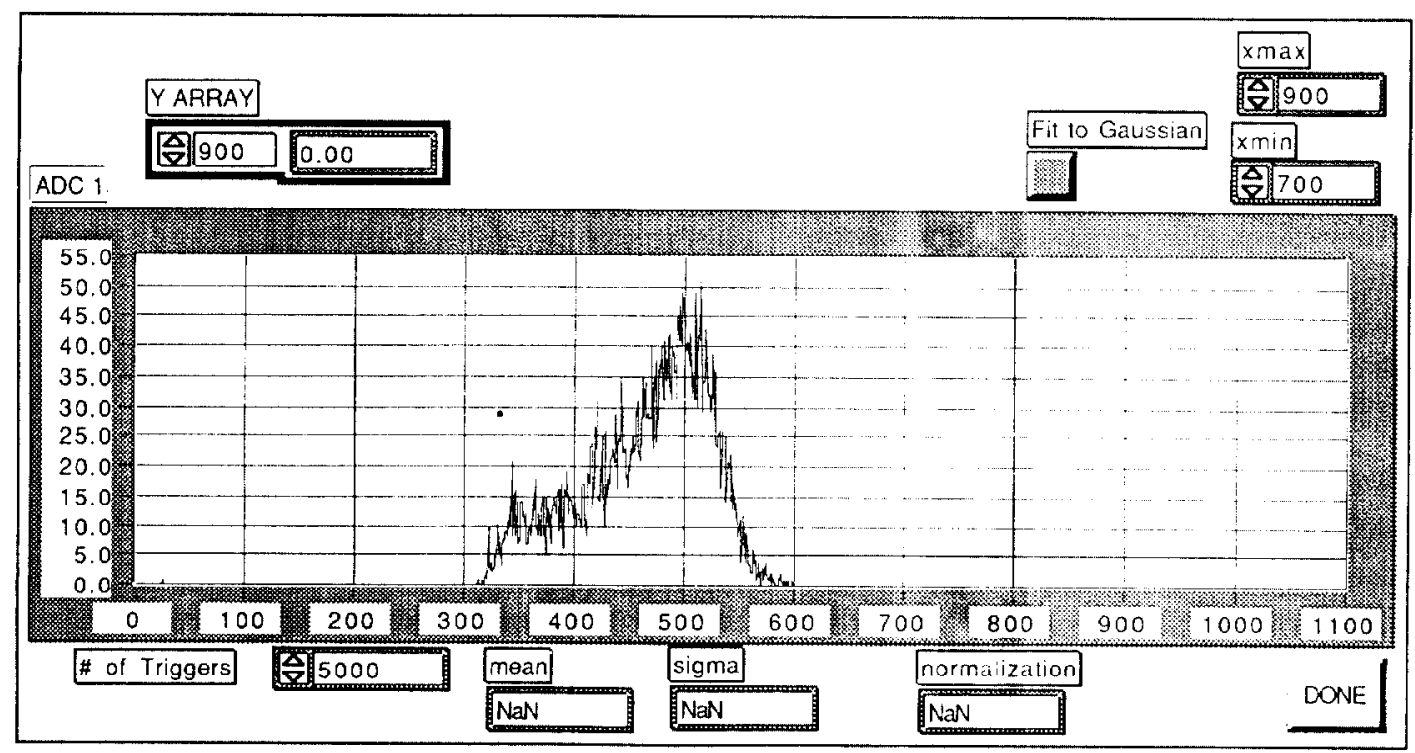

Figure 3. Online data for $300 \mathrm{MeV} /$ nucleon Au going through a fiber in the central region of the protolype detector. The PMT high voltage was $\mathrm{HV}=-2000 \mathrm{~V}$. 


\section{Transmission Calculations}

In order to assess whether or not one can obtain sufficient dynamic range with these fibers to make a good halo detector, as well as whether an image intensifier would be needed for use with a $C C D$, tests were made comparing the response with a heavy beam to that of an electron source, and estimates were made of the transmission of light from the particle passing through the fiber to the PMT. Figure 4 shows a schematic of the transmission of the light from the fiber to the PMT, with the expected losses noted. An additional complication is the fact that the scintillation mechanism for heavy ions is different than that for electrons, and exhibit a $Z$ dependence. This can be estimated from earlier studies of the response of plastic scintillators to heavy ions [6], if the assumption is made that the response is to first order independent of the particular scintillator material.

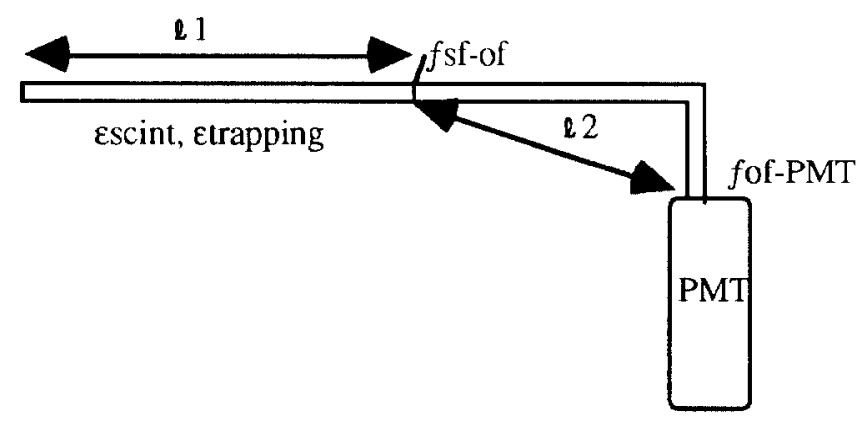

Figure 4. Schematic of transmission losses in fiber detector

The measured light output of the detector, $\mathrm{L}_{\mathrm{d}}$, then, is given by the light output of the scintillating fiber for a heavy ion, $\mathrm{L}_{\mathrm{HI}}$, multiplied by the various transmission loss factors, multiplied by the gain of the PMT:

$$
\begin{aligned}
& \mathrm{LD}_{\mathrm{b}}=\mathrm{LHII}^{*} \varepsilon_{\text {trapping }} *\left(1-\mathrm{A}_{\mathbf{l} 1}\right) * f_{\mathrm{SF}} \cdot \text { of } *\left(1-\mathrm{A}_{\mathbf{2} 2}\right) \\
& \text { * } f_{\text {of }-\mathrm{PMT}} * \mathrm{GPMT}
\end{aligned}
$$

where $\varepsilon_{\text {trapping }}$ is the percentage of total light piped down the fiber. $A_{\ell} 1$ and $A_{\ell} 2$ are the attenuation factors for the lengths of the scintillating fiber and optical fiber, respectively, $f_{\text {sf-of }}$, is the fraction of light transmitted through the scintillating fiber/optical fiber interface, $f_{\text {of-PMT, }}$, is the fraction of light transmitted from the optical fiber to the PMT, and GPMT is the gain of the PMT. $\varepsilon_{\text {trapping, }} A_{\ell} 1$, and $A_{\ell} 2$ are properties of the fibers and are given in the data sheet from Bicron Corp. [2] $f_{\text {St-of, }} f_{\text {of-PMT, }}$ and GPMT can be estimated or measured using an electron source.

An attempt was made to measure directly the transmission to the PMT using a $1 \mathrm{MeV}$ electron source; unfortunately, the losses are large enough so that the signal is in the noise by the time it reaches the PMT. (This immediately implies that this detector is limited to particles heavier than protons, where the energy loss is greater than 1 $\mathrm{MeV}$. It will probably also not work with helium, since the energy loss from Figure 1 is around $1 \mathrm{MeV}$, and the light output is decreased for a heavy ion relative to an electron of the same energy loss. [7] )

An estimate of the transmission from Eqn. 1 can be made, knowing the attenuation lengths $A_{\ell} 1$ and $A_{\ell} 2$ are equal and given by the manufacturer as 2 meters for a $1 / \mathrm{e}$ drop in light, and $\varepsilon_{\text {trapping }}$ is given by the manufacturer as $3.4 \%$ (it may be higher since one end of the fiber was made reflective). The scintillation efficiency of the fiber is quoted by Bicron to be $2.8 \%$ for electrons or minimum-ionizing particles, that is, $2.8 \%$ of the energy is converted into light. The scintillation photons have an average energy of $3 \mathrm{eV}$. [2] Therefore with a $1 \mathrm{MeV}$ electron, $\approx 9000$ photons are made, of which 350 make it to the end of the fiber. $\ell_{1}+\ell_{2}$ was approximately 0.5 meters, so the attenuation in the fiber is small. The coupling between the scintillating fibers and optical fibers should be near $100 \%$ efficient as the two sets of fibers are well matched in size and composition. So we can estimate that 300 photons, or $900 \mathrm{eV}$ of light make it through the fibers. The coupling to the PMT was not good for these tests, as the PMTs were much larger than is optimum to couple to a 1 $\mathrm{mm}$. fiber. Coupling the fibers to a CCD should be more efficient. For Au at $300 \mathrm{MeV} / \mathrm{u}$, the energy loss in the fiber is $3000 \mathrm{MeV}$, so $\approx 10^{6}$ photons should make it to the PMT., not taking into account decrease in light output for Au relative to electrons, which isn't known at these energies.

\section{Conclusion}

A prototype scintillating fiber detector was built and tested with heavy ions at the Bevalac. In addition to the $\mathrm{Au}$ beam results shown here, the detector was used to look at I a and Ne beams. These beams were easily seen with the detector, but because of efficiency problems, one detector will not cover the full range of ions and energies run at the Bevalac. A second detector could be made with thicker fibers for the light ions. This detector appears to be a useful device for this application. The use of CCD readouts rather than PMTs would make it more portable and straightforward to use. An image intensifier would probably be necessary in the halo regions (not the central beam regions) to obtain the dynamic range that is desirable.

\section{References}

[1] A.J. Davis, ct al., "Scintillating Optical Fiber Trajectory Detectors", Nucl. Instr. and Methods A276, 347 (1989).

[2] Bicron Corporation, Newsbury, Ohio

[3] TM Apple Computer, Inc., Cupertino, CA

[4] TM National Instruments, Inc., Austin, TX

[5] M. LeBlanc, et. al., "SOFI: A Scintillating Optical Fiber Imager", Nucl. Instr. and Methods A273, 583 (1988).

[5] M.A. McMahan, "The Response of Scintillators to Heavy Ions - I. Plastics", IEEE Trans. an Nucl. Sci. 35, 42(1988).

[6] J.B. Birks, "Theory and Practice of Scintillation Counting", (Pergamon, Oxford, 1964).

This work was supported by the Director, Office of High Energy Research, Division of Nuclear Physics of the U.S. Department of Energy under Contract DE-AC03-76SF00098. 